\title{
Anisotropic wave-equation traveltime and waveform inversion
}

\section{Shihang Feng* and Gerard T. Schuster, King Abdullah University of Science and Technology (KAUST)}

\section{SUMMARY}

The wave-equation traveltime and waveform inversion (WTW) methodology is developed to invert for anisotropic parameters in a vertical transverse isotropic (VTI) meidum. The simultaneous inversion of anisotropic parameters $v_{p 0}, \varepsilon$ and $\delta$ is initially performed using the wave-equation traveltime inversion (WT) method. The WT tomograms are then used as starting background models for VTI full waveform inversion. Preliminary numerical tests on synthetic data demonstrate the feasibility of this method for multi-parameter inversion.

\section{INTRODUCTION}

Conventional FWI inverts for a velocity model by using an objective function that minimizes the $\mathrm{L} 2$ norm of the residuals between the predicted and the observed traces (Tarantola, 2005). However, such a misfit function is highly non-linear and the iterations often get stuck in a local minimum. In order to mitigate this problem, a skeletonized representation of the data such as traveltimes (Luo and Schuster, 1991a,b) can be inverted to obtain the low-intermediate wavenumber details of the background velocity model. The misfit function for such skeletonized inversion is quasi-linear and enjoys better convergence properties than conventional full waveform inversion.

WT was previously implemented under the isotropic approximation (Zhou et al., 1995, 1997; Van Leeuwen and Mulder, 2010). However, the first arrivals can be strongly influenced by anisotropy in the near-surface. The consequence is that isotropic migration will lead to mis-positioning and defocusing of reflectors in depth (Shen et al., 2012). Thus, it is necessary to take anisotropy into account when using WT on near-surface data.

In this paper, we extend the WT methodology to anisotropic media. The gradients are obtained by smearing the traveltime residuals along the anisotropic wavepaths. However, for multicomponent inversion, the problem is highly under-determined and the solution is not unique. Therefore, the full-waveform inversion (FWI) method is combined with WT starting models for waveform inversion of anisotropic data.

The first section of this paper presents the theory. In the second part, a sensitivity analysis of anisotropic parameters is presented. Numerical tests on synthetic data are shown in the third section and the conclusions are in the last section.

\section{THEORY}

\section{Anisotropic Wave-Equation Traveltime Inversion}

WT can be extended to an anisotropic medium by inverting for the anisotropy parameters $v_{p 0}, \varepsilon$ and $\delta$ using the traveltime misfit function,

$$
\varepsilon=\frac{1}{2} \sum_{s, g}[\Delta \tau(\mathbf{g}, \mathbf{s})]^{2},
$$

where $\Delta \tau(\mathbf{g}, \mathbf{s})=\tau_{\text {obs }}(\mathbf{g}, \mathbf{s})-\tau_{c a l}(\mathbf{g}, \mathbf{s})$ is the traveltime residual.

The anisotropic parameters $\mathbf{m}(\mathbf{x})$ can be estimated using any gradient-based method. To update the anisotropic parameters, the steepest descent method gives

$$
\mathbf{m}(\mathbf{x})^{(i+1)}=\mathbf{m}(\mathbf{x})^{(i)}-\alpha \sum_{s, g} \Delta \tau(\mathbf{g}, \mathbf{s}) \frac{\partial \tau_{c a l}(\mathbf{g}, \mathbf{s})}{\partial \mathbf{m}(\mathbf{x})},
$$

where $\alpha$ is the step length and $i$ is the iteration index.

The Fréchet derivative $\frac{\partial \tau_{c a l}(\mathbf{g}, \mathbf{s})}{\partial \mathbf{m}(\mathbf{x})}$ can be obtained using the implicit function theorem (Luo and Schuster, 1991a) as

$$
\frac{\partial \tau_{c a l}}{\partial \mathbf{m}}=-\frac{\partial \dot{\Phi} / \partial \mathbf{m}}{\partial \dot{\Phi} / \partial \tau_{c a l}},
$$

where $\dot{\Phi}$ is the time derivative of a crosscorrelation function between the predicted $p(\mathbf{g}, t+\Delta \tau \mid \mathbf{s}, 0)$ and the observed data $p(\mathbf{g}, t \mid \mathbf{s}, 0)^{\text {obs }}$,

$$
\dot{\Phi}(\mathbf{s}, \mathbf{g}, \Delta \tau))=\int_{0}^{T} d t p(\mathbf{g}, t \mid \mathbf{s}, 0)^{o b s} \dot{p}(\mathbf{g}, t+\Delta \tau \mid \mathbf{s}, 0)=0 .
$$

The decoupled $\mathrm{P}$-wave equation for vertical transverse isotropic (VTI) media in the time and wavenumber domain is given by (Zhan et al., 2012)

$$
\frac{1}{v_{p_{0}}^{2}} \frac{\partial^{2} P}{\partial t^{2}}=-\left[(1+2 \varepsilon) k_{x}^{2}+k_{z}^{2}-\frac{2(\varepsilon-\delta) k_{x}^{2} k_{z}^{2}}{k_{x}^{2}+k_{z}^{2}}\right] P,
$$

where $k_{x}, k_{z}$ are spatial wavenumbers and $P$ is the wavefield function in the time-wavenumber domain.

Substituting the Fréchet derivatives into equation 2 yields the traveltime misfit gradients for the three anisotropy parameters $v_{p 0}, \varepsilon$ and $\delta$ :

$$
\begin{aligned}
\gamma_{v_{p 0}}^{\tau}(\mathbf{x}) & =\sum_{s, g} \int_{0}^{T} d t\left[\frac{2}{v_{p 0}^{3}(\mathbf{x})} \dot{g}(\mathbf{x}, t \mid \mathbf{g}, 0) \star \dot{p}(\mathbf{x}, t \mid \mathbf{s}, 0)\right] \\
& \times \Delta \tilde{\tau}(\mathbf{g}, t \mid \mathbf{s}, 0), \\
\gamma_{\varepsilon}^{\tau}(\mathbf{x}) & =\sum_{s, g} \int_{0}^{T} d t[g(\mathbf{x}, t \mid \mathbf{g}, 0) \star U(\mathbf{x}, t \mid \mathbf{s}, 0)] \\
& \times \Delta \tilde{\tau}(\mathbf{g}, t \mid \mathbf{s}, 0),
\end{aligned}
$$

$$
\begin{aligned}
\gamma_{\delta}^{\tau}(\mathbf{x}) & =\sum_{s, g} \int_{0}^{T} d t[g(\mathbf{x}, t \mid \mathbf{g}, 0) \star V(\mathbf{x}, t \mid \mathbf{s}, 0)] \\
& \times \Delta \tilde{\tau}(\mathbf{g}, t \mid \mathbf{s}, 0),
\end{aligned}
$$




\section{Anisotropic WTW inversion}

where $U(\mathbf{x}, t \mid \mathbf{s}, 0)$ and $V(\mathbf{x}, t \mid \mathbf{s}, 0)$ are given as

$$
\begin{aligned}
& U(\mathbf{x}, t \mid \mathbf{s}, 0)=-2 \mathscr{F}^{-1}\left(\frac{k_{x}^{4}}{k_{x}^{2}+k_{z}^{2}}\right) * p(\mathbf{x}, t \mid \mathbf{s}, 0), \\
& V(\mathbf{x}, t \mid \mathbf{s}, 0)=-2 \mathscr{F}^{-1}\left(\frac{k_{x}^{2} k_{z}^{2}}{k_{x}^{2}+k_{z}^{2}}\right) * p(\mathbf{x}, t \mid \mathbf{s}, 0),
\end{aligned}
$$

where $\star$ represents temporal convolution, $*$ represents spatial convolution and $\Delta \tilde{\tau}$ is the pseudo-traveltime residual

$$
\Delta \tilde{\tau}(\mathbf{g}, t \mid \mathbf{s}, 0)=-\frac{1}{E} \dot{p}(\mathbf{g}, t-\Delta \tau \mid \mathbf{s}, 0)^{o b s} \Delta \tau(\mathbf{g}, t \mid \mathbf{s}, 0),
$$

which is the time-shifted recorded data weighted by the associated traveltime residual.

Anisotropic Wave-Equation Traveltime and Waveform Inversion

The wave-equation traveltime and waveform inversion (WTW) misfit function is a blend of WT and FWI misfit functions

$$
\varepsilon=\frac{1-a}{2} \sum_{s, g}[\Delta \tau(\mathbf{g}, \mathbf{s})]^{2}+\frac{a}{2} \sum_{s, g, t}[\Delta d(\mathbf{g}, \mathbf{s}, t)]^{2},
$$

where $\Delta d(\mathbf{g}, \mathbf{s}, t)=d_{o b s}(\mathbf{g}, \mathbf{s}, t)-d_{c a l}(\mathbf{g}, \mathbf{s}, t)$ is the data residual, $a$ is a weighting function used to adjust the weights of the two misfit functions and $a$ can be gradually varied from 0 to 1 as the iterations proceed.

The WTW hybird gradient is given as

$$
\gamma(\mathbf{x})=(1-a) \gamma^{\tau}(\mathbf{x})+a \gamma^{d}(\mathbf{x}),
$$

where $a$ is set to 0 for the early iterations of the waveform inversion to invert for the low-intermediate wavenumber of the model with traveltime inversion. The details of the background models are reconstructed in the latter iterations using waveform inversion by setting $a=1$ (Zhou et al., 1997).

\section{SENSITIVITY ANALYSIS}

In Figure 1, the common shot gathers (CSGs) from two-layered anisotropic and isotropic models are shown. The first arrivals are indicated by the red lines, where the time shifts of first arrivals caused by anisotropic effect will lead to incorrect velocity models inverted by an isotropic tomography method. Thus, It is important to study how anisotropy affects the kinematics of the data. Here the sensitivity analysis is computed using the Thomsen parameters $v_{p 0}, \varepsilon, \delta$ (Thomsen, 1986).

Transforming forward modeling equation into the frequency domain and using the asymptotic Green's function and $\mathbf{p}$ which is the unitary vector related the wavenumber vector by $\mathbf{k}=$ $\frac{\omega}{v_{p 0}} \mathbf{p}$ (Alkhalifah and Plessix, 2014), we can get

$$
r_{p}(\mathbf{s}, \mathbf{g}, \omega)=-\omega^{2} s(\omega) \int_{V} A\left(\mathbf{s}, \mathbf{x}^{\prime}, \mathbf{g}, \omega\right) \mathbf{a}\left(\mathbf{x}^{\prime}\right) \cdot \mathbf{r}\left(\mathbf{x}^{\prime}\right) d x^{\prime 3},
$$

where

$$
A\left(\mathbf{s}, \mathbf{x}^{\prime}, \mathbf{g}, \omega\right)=\frac{G\left(\mathbf{s}, \mathbf{x}^{\prime}, \omega\right) G\left(\mathbf{g}, \mathbf{x}^{\prime}, \omega\right)}{v_{p 0}^{2}\left(\mathbf{x}^{\prime}\right)}
$$
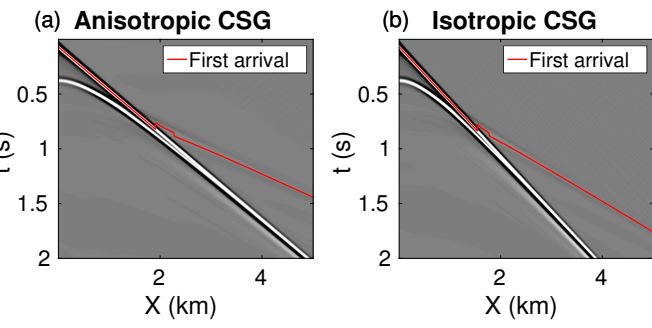

Figure 1: CSGs associated with a) anisotropic and b) isotropic two-layer models. (The red lines indicate the first arrivals).

and

$$
\mathbf{r}=\left(\begin{array}{c}
r_{v_{p 0}} \\
r_{\varepsilon} \\
r_{\delta}
\end{array}\right) ; \mathbf{a}=\left(\begin{array}{c}
2 \\
-2 p_{s x}^{4} \\
-2 p_{s x}^{2} p_{s z}^{2}
\end{array}\right)
$$

The vector a represents the radiation patterns for each anisotropic parameter $v_{p 0}, \varepsilon$ and $\delta$ (Aki and Richards, 1980), and $\mathbf{p}_{s}$ is expressed with the source incidence angle $\theta / 2$ :

$$
\mathbf{p}_{s}=\left(\begin{array}{c}
p_{s x} \\
p_{s z}
\end{array}\right)=\left(\begin{array}{c}
\sin (\theta / 2) \\
\cos (\theta / 2)
\end{array}\right) .
$$

Figure 2 shows the radiation patterns for the anisotropic parameters $v_{p 0}, \varepsilon$ and $\delta$ for a horizontal reflector. The radiation pattern of $v_{p 0}$ is independent of the angle which is similar to the isotropic case. The perturbation in $\varepsilon$ is most sensitive to the large scattering angle, thus large offsets are important for $\varepsilon$ inversion. However, there is a trade-off between $v_{p 0}$ and $\varepsilon$ at large angles. For $\delta$, the trade-off would be maximum with the incidence angle at $45^{\circ}$.

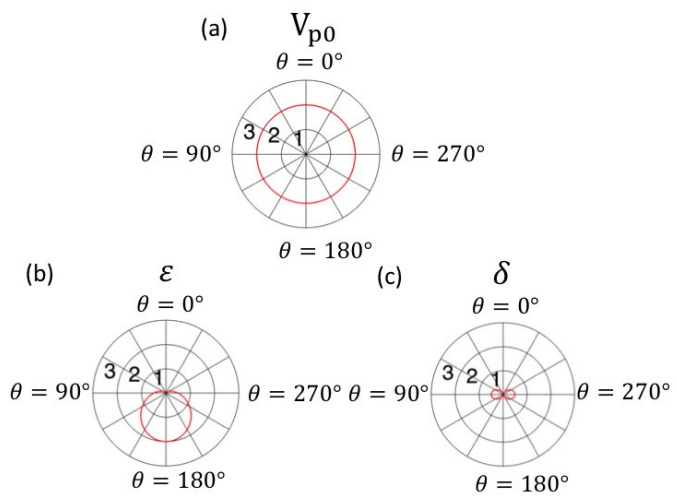

Figure 2: The radiation pattern of a) $v_{p 0}$, b) $\varepsilon$ and c) $\delta$ for a horizontal reflector.

The two-layered anisotropic model shown in Figure 3 is used to test the influence of anisotropic parameters on traveltimes. The horizontal reflector is buried at $1.25 \mathrm{~km}$ in depth and the offset from source to receiver is $2.5 \mathrm{~km}$. Two events can be observed at the receiver. The left one in Figure 4 is the transmission wave with a scattering angle of $180^{\circ}$ and the right one 


\section{Anisotropic WTW inversion}

results from the reflection wave with a scattering angle of $90^{\circ}$ (Figure 3).

The blue line in Figure 4 is the signal with the original model in Figure 3. An increase of $10 \%$ relative to each anisotropic parameter is applied through the whole model and the red line in Figure 4 is the response after the change. The sensitivty of each parameter with respect to traveltime and scattering angle can be seen based on the time shift caused by the change of anisotropic parameters.

According to the radiation pattern, $v_{p 0}$ is sensitive to all angles. So, the two events have clear time shifts and the reflection event shifts more due to a longer wavepath. For the change of $\varepsilon$, the transmission travetime is more sensitive than the reflection. This is in agreement with the $\varepsilon$ radiation pattern. When changing $\delta$, the two events have a minor time shift due to their weak sensitivity to changes in $\delta$.

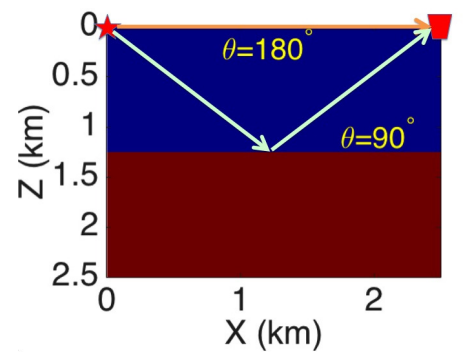

Figure 3: The two layered model used for the sensitivity analysis of anisotropic parameters. (Upper layer: $v_{p 0}=2000 \mathrm{~m} / \mathrm{s}$, $\varepsilon=0.3, \delta=0.25$; lower layer: $v_{p 0}=2500 \mathrm{~m} / \mathrm{s}, \varepsilon=0.35$, $\delta=0.3$.)
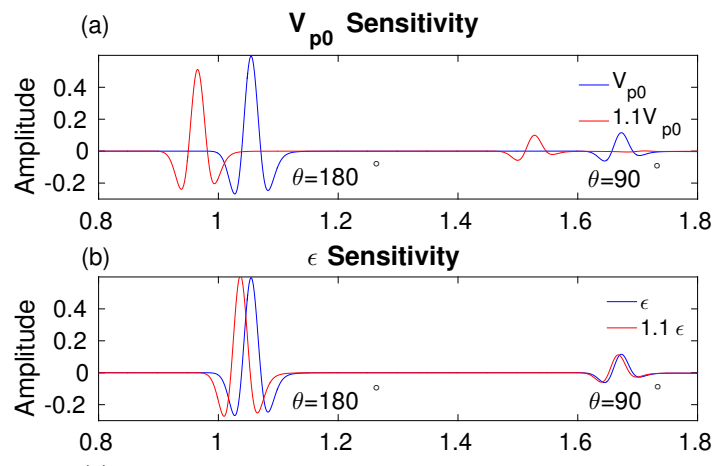

(c)

$\delta$ Sensitivity

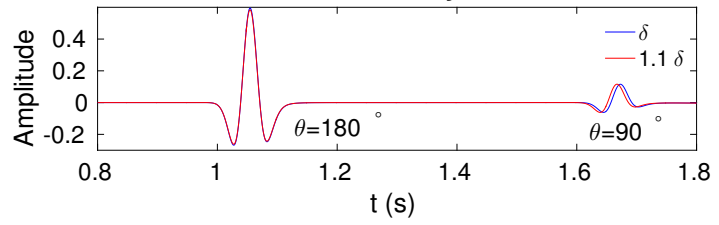

Figure 4: The transmission $\left(\theta=180^{\circ}\right)$ and reflection wave $\left(\theta=90^{\circ}\right)$ are time shifted with respect to changes in a) $v_{p 0}$, b) $\varepsilon$ and c) $\delta$.

\section{NUMERICAL TEST}

Anisotropic WT and WTW are now applied to synthetic data. The observed data are simulated by the rapid expansion method (REM) (Pestana et al., 2011) based on the pure P-wave VTI wave-equation.

In test 1 , the models for the anisotropic parameters are shown in Figures 5(a), 5(b) and 5(c). The model size is $750 \mathrm{~m}$ in the $\mathrm{X}$ direction and $250 \mathrm{~m}$ in the $\mathrm{Z}$ direction with a grid spacing of $6.25 \mathrm{~m}$. Fifty-nine sources are located on the surface with a spacing of $12.5 \mathrm{~m}$. The data are recorded by 120 receivers spaced at an interval of $6.25 \mathrm{~m}$. The source wavelet is a Ricker wavelet with a peak frequency of $30 \mathrm{~Hz}$. The three parameters $v_{p 0}, \varepsilon$ and $\delta$ are inverted simultaneously. The anisotropic WT becomes a highly under-determined system since only traveltime information is used. The tomograms obtained after 10 iterations are shown in Figures 5(g), 5(h) and 5(i). The shape of the anticline is not inverted and the three layers are roughly imaged. To reduce the non-uniqueness problem, we adapt WTW to the anisotropic data inversion. After 10 iterations, the WT gradient in equation 10 is turned off by setting $a=0$ and the waveform gradient is used to give the WTW inversion result in Figures 5(j), 5(k) and 5(1). We notice that WTW shows more accurate tomograms compared to WT. Both the $v_{p 0}$ and $\varepsilon$ tomograms are consistent with the true models. However, the $\delta$ results can't be recovered accurately due to its low sensitivity to changes in $\delta$.

In test 2, WTW methodology is applied to the Marmousi model shown in Figures 6(a), 6(b) and 6(c). The acquisition geometry on the top surface comprises 300 fixed receivers with a spacing of $20 \mathrm{~m}$ and 150 sources with a spacing of $40 \mathrm{~m}$. The initial models are horizontal layered with anisotropic parameters linearly increasing in depth. The $\varepsilon$ and $\delta$ in the deep part can't be updated by WT as shown in Figures 6(h) and 6(i) due to their low sensitivity to the changes in $\varepsilon$ and $\delta$. As a consequence, the $\varepsilon$ and $\delta$ can't been inverted well by WTW as shown in Figures $6(\mathrm{k})$ and $6(\mathrm{l})$. Only the $v_{p 0}$ is inverted accurately.

\section{CONCLUSIONS}

Wave-equation traveltime inversion (WT) can be used to invert for the anisotropic parameters from anisotropic data. However, the solution is not unique when inverting for all the anisotropic parameters simultaneously. In order to solve this problem, anisotropic WTW is applied to the data since it takes the waveform information into account. Synthetic tests show that the accuracy of the anisotropic tomograms can be improved by combining wave-equation traveltime inversion with waveform inversion.

\section{ACKNOWLEDGMENTS}

The research reported in this publication is supported by funding from King Abdullah University of Science and Technology (KAUST). We also thank the sponsors for supporting the Consortium of Subsurface Imaging and Fluid Modeling (CSIM). 


\section{Anisotropic WTW inversion}

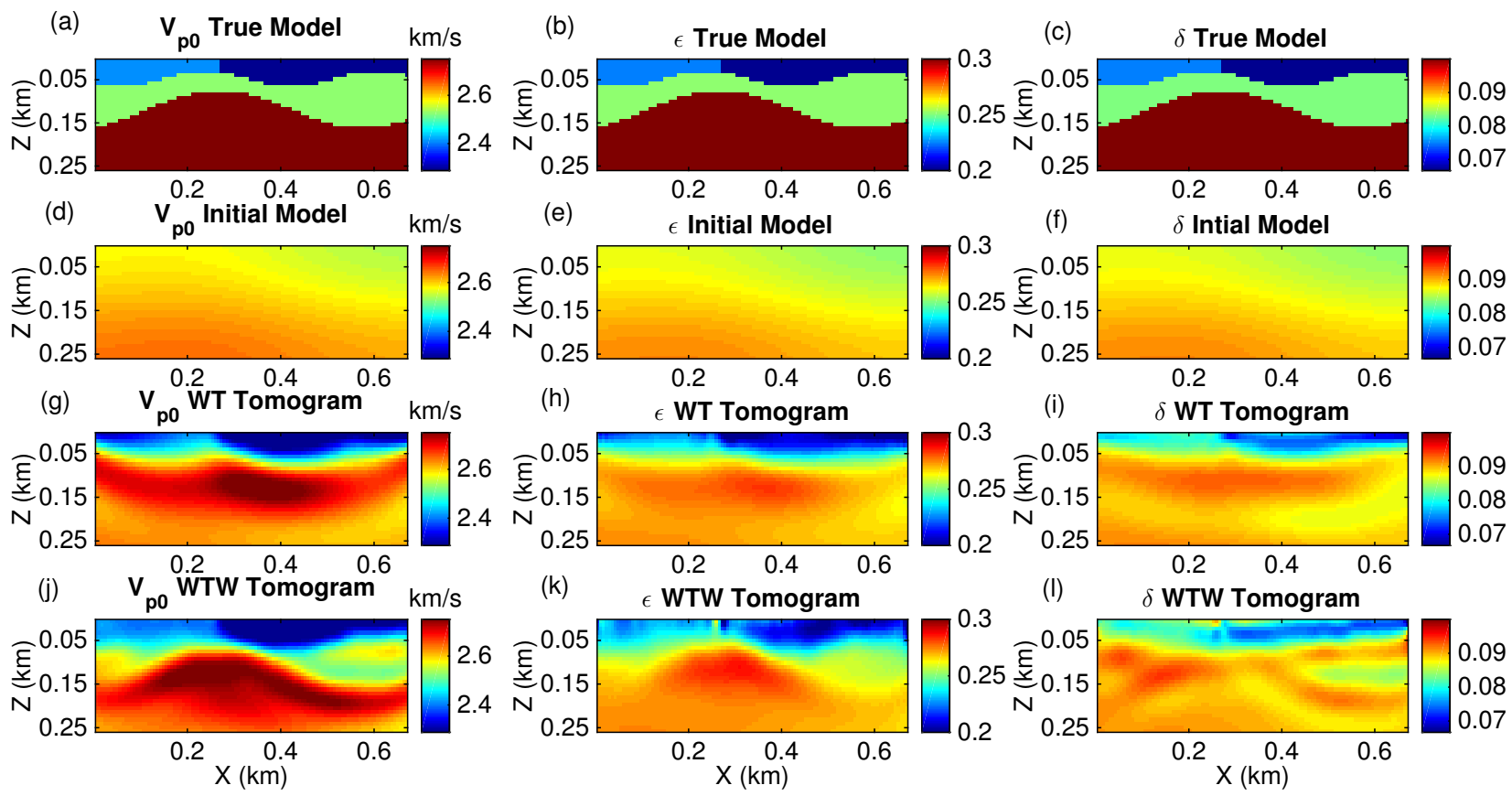

Figure 5: Synthetic test 1: (a), (b), (c) The true $v_{p 0}, \varepsilon$ and $\delta$ models ; (d), (e), (f) the initial $v_{p 0}, \varepsilon$ and $\delta$ model; (g), (h), (i) the $v_{p 0}$, $\varepsilon$ and $\delta$ tomograms after 10 iterations using WT; (j), (k), (1) the $v_{p 0}, \varepsilon$ and $\delta$ tomograms using 30 iterations of WTW.
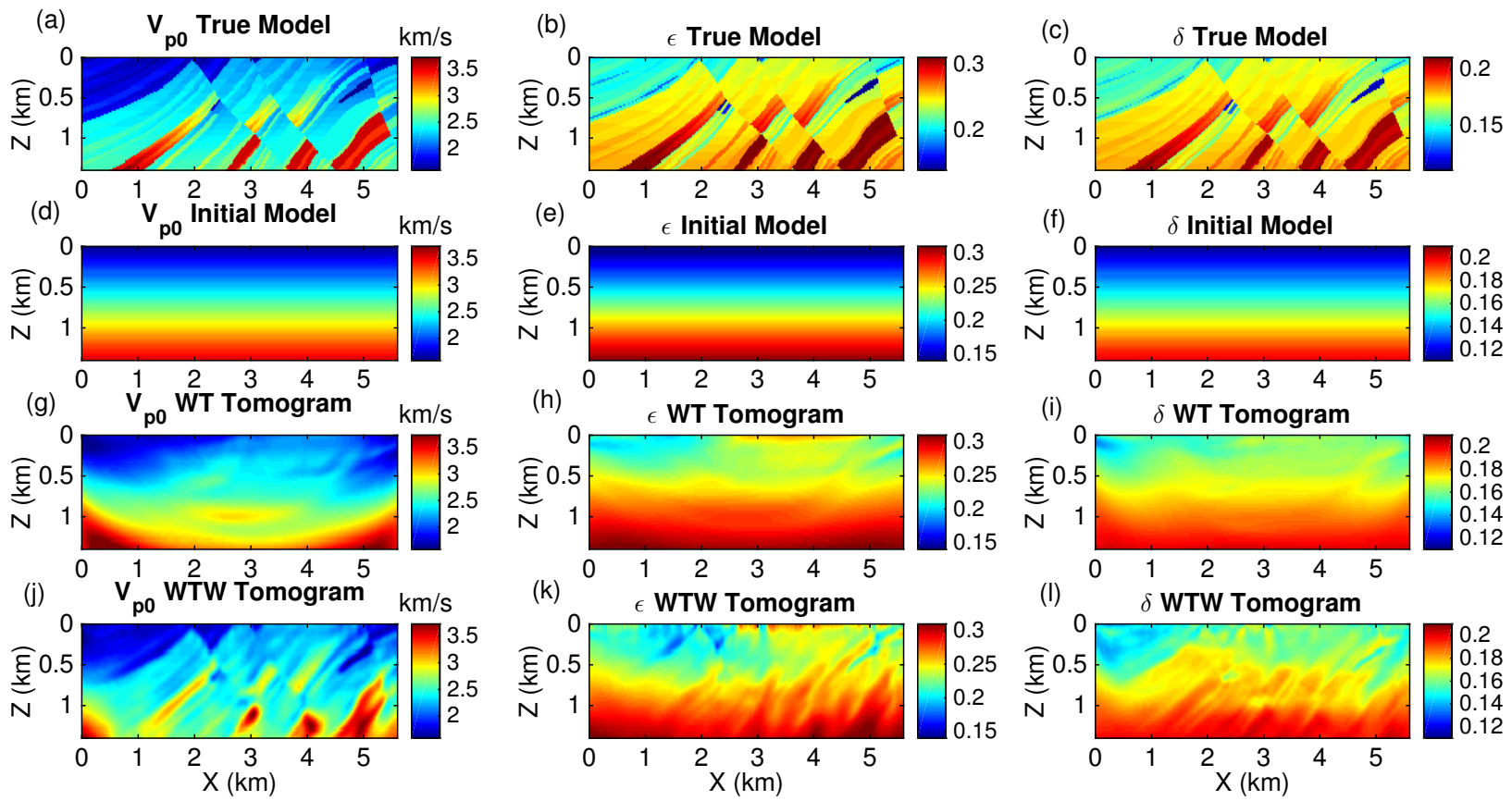

Figure 6: Synthetic test 2: (a), (b), (c) The true $v_{p 0}, \varepsilon$ and $\delta$ models ; (d), (e), (f) the initial $v_{p 0}, \varepsilon$ and $\delta$ model; (g), (h), (i) the $v_{p 0}$, $\varepsilon$ and $\delta$ tomograms after 10 iterations using WT; (j), (k), (l) the $v_{p 0}, \varepsilon$ and $\delta$ tomograms using 30 iterations of WTW. 
EDITED REFERENCES

Note: This reference list is a copyedited version of the reference list submitted by the author. Reference lists for the 2016 SEG Technical Program Expanded Abstracts have been copyedited so that references provided with the online metadata for each paper will achieve a high degree of linking to cited sources that appear on the Web.

\section{REFERENCES}

Aki, K., and P. G. Richards, 1980, Quantitative seismology, 2nd ed.: University Science Books.

Alkhalifah, T., and R.-É. Plessix, 2014, Arecipeforpractical full-waveform inversion in anisotropic media: An analytical parameter resolution study: Geophysics, 79, no. 3, R91-R101, http://dx.doi.org/10.1190/geo2013-0366.1.

Luo, Y., and G. T. Schuster, 1991a, Wave equation inversion of skeletalized geophysical data: Geophysical Journal International, 105, 289-294, http://dx.doi.org/10.1111/j.1365246X.1991.tb06713.x.

Luo, Y., and G. T. Schuster, 1991b, Wave-equation traveltime inversion: Geophysics, 56, 645-653, http://dx.doi.org/10.1190/1.1443081.

Pestana, R. C., B. Ursin, and P. L. Stoffa, 2011, Separate p-and sv-wave equations for VTI media: Presented at the 12th International Congress of the Brazilian Geophysical Society, http://dx.doi.org/10.1190/sbgf2011-253.

Shen, X., 2012, Early-arrival waveform inversion for near-surface velocity and anisotropic parameter: Parameterization study: 82nd Annual International Meeting, SEG, Expanded Abstracts, http://dx.doi.org/10.1190/segam2012-0730.1.

Tarantola, A., 2005, Inverse problem theory and methods for model parameter estimation: Siam. http://dx.doi.org/10.1137/1.9780898717921.

Thomsen, L., 1986, Weak elastic anisotropy: Geophysics, 51, 1954-1966, http://dx.doi.org/10.1190/1.1442051.

Van Leeuwen, T., and W. Mulder, 2010, A correlation-based misfit criterion for wave-equation traveltime tomography: Geophysical Journal International, 182, 1383-1394, http://dx.doi.org/10.1111/j.1365-246X.2010.04681.x.

Zhan, G., R. C. Pestana, and P. L. Stoffa, 2012, Decoupled equations for reverse time migration in tilted transversely isotropic media: Geophysics, 77, no. 2, T37-T45, http://dx.doi.org/10.1190/geo20110175.1.

Zhou, C., W. Cai, Y. Luo, G. T. Schuster, and S. Hassanzadeh, 1995, Acoustic wave-equation traveltime and waveform inversion of crosshole seismic data: Geophysics, 60, 765-773, http://dx.doi.org/10.1190/1.1443815.

Zhou, C., G. T. Schuster, S. Hassanzadeh, and J. M. Harris, 1997, Elastic wave equation traveltime and waveform inversion of crosswell data: Geophysics, 62, 853-868, http://dx.doi.org/10.1190/1.1444194. 